\title{
Role of the pathologist in the diagnosis of hereditary non-polyposis colorectal cancer
}

\author{
Jeremy R. Jass* \\ McGill University, Montreal, Quebec, Canada
}

\begin{abstract}
The aim of this paper is to indicate how the pathologist may suspect a diagnosis of hereditary non-polyposis colorectal cancer (HNPCC) on the basis of histological criteria and patient age alone. A single morphological feature, namely the presence of intra-epithelial lymphocytes (tumor infiltrating lymphocytes), identifies the majority of colorectal cancers (CRC) with the DNA microsatellite instability-high phenotype. A number of pathological criteria can help to distinguish HNPCC from sporadic MSI-H CRC, though age below 60 years is an important pointer towards HNPCC. Immunohistochemistry to demonstrate loss of expression of DNA mismatch repair genes serves as a highly reliable test of mismatch repair deficiency if antibodies to hMLH1, hMSH2, hMSH6 and hPMS2 are employed.
\end{abstract}

Keywords: HNPCC, pathology, diagnosis, tumor infiltrating lymphocytes

\section{Historical perspectives}

In a number of respects it is ironic that the earliest records of hereditary non-polyposis colorectal cancer (HNPCC) can be traced back to the work of a pathologist (Aldred Warthin) [1]. Pathologists do not normally interact directly with patients or their families. Familial disorders, including cancer family syndromes, are often associated with dysmorphic or cutaneous manifestations that may be recognized by physicians such as geneticists or dermatologists. However, when the phenotype includes uncommon or unusual tissue alterations affecting different internal organs, the pathologist is in a position to deduce that the seemingly unrelated features are in fact the result of an inherited syndrome. Examples of familial cancer syndromes first recognized by pathologists are multiple endocrine neoplasia and the Beckwith-Wiedemann syndrome featuring such rare types of tumor as medullary carcinoma of the thyroid and renal nephroblastoma respectively [2,3].

* Professor J.R. Jass, Department of Pathology, McGill University, Duff Medical Building, 3775 University Street, Montreal, Quebec, Canada H3A 2B4. Tel.: +1 514398 7192; Fax: +1 514398 7446; E-mail: jeremy.jass@mcgill.ca.
In the case of HNPCC the internal malignancies are neither uncommon nor especially unusual in terms of their histological appearances. Nevertheless, Warthin developed the practice of linking a histopathological diagnosis with a family history and even conducted his work on samples that were representative of the general population of the State of Michigan. He also had the advantage of the personal knowledge of an individual, his seamstress, who correctly predicted her early death from an internal malignancy. A measure or irony may also be attached to the fact that when Warthin first documented 'family G', shown many years later to carry a DNA mismatch repair gene, colonic cancer was not well represented among the internal malignancies [4]. The latter comprised gastric cancers (seven cases) and endometrial cancer (ten cases). The final irony was that over 80 years were to pass before other pathologists were to demonstrate any interest in HNPCC. Despite the dogged determination of Lynch to thoroughly document the clinical features of HNPCC and thereby highlight its reality as a clinical entity [5-7], HNPCC was overshadowed by a different form of hereditary colon cancer - familial adenomatous polyposis (FAP). FAP is rarer than HNPCC, but is associated with more obvious and unusual tissue manifestations in terms of hun- 
dreds if not thousands of colonic polyps, fibromatosis or desmoid disease, as well as colorectal cancer (CRC).

In the following, it will be shown that $\mathrm{CRC}$ in $\mathrm{HN}$ $\mathrm{PCC}$ is not identical with the common forms of large bowel malignancy, but may be distinguished on the basis of morphological and immunohistochemical features. The pathologist is now in a position to suspect a diagnosis of HNPCC on the basis of histological findings alone.

\section{Morphology of colorectal cancer in HNPCC}

A review of 140 colorectal cancers derived from members of 34 families that fulfilled the Amsterdam criteria showed a greater than expected proportion of cases with mucinous differentiation (19\%) and poor differentiation $(39 \%)$. A circumscribed or pushing invasive tumor margin and peri-tumoral lymphocytic infiltration were not over-represented. However 13 (9\%) of the cancers were described as poorly differentiated with a circumscribed margin and marked lymphocytic infiltration or as 'medullary' [8,9]. CRC with this combination of features and showing an unexpectedly favorable clinical outcome had been described previously, including in young subjects [10]. These tumors have also been described as 'lymphoepithelioma-like' and may arise within the dome or M-cell epithelium that overlies mucosa associated lymphoid tissue $[11,12]$. While it was interesting that HNPCC cancers showed histological differences from usual CRC, it was concluded that the presence of such features was less important than family history or age at onset for reaching a diagnosis of HNPCC [8].

Following the recognition of the mutator phenotype [13], families meeting the Amsterdam criteria were grouped according to whether CRC were DNA microsatellite instability-high (MSI-H) or not [14]. Cancers that came from members of Amsterdam families in which two or more cancers were found to be MSI-H showed the clinical and pathological features that have come to be associated with HNPCC: (1) proximal anatomic location, (2) multiple colorectal cancers, (3) poor differentation (including medullary pattern), (4) mucinous differentiation, (5) lower frequency of lymph node metastasis, and (6) lower frequency of DNA aneuploidy [14]. By contrast, these features were not over-represented in Amsterdam positive families in which CRC were microsatellite stable (MSS). By this stage, many clinicians and scientists were beginning to equate HNPCC with a specific syndrome caused by the inheritance of a germline mutation in one of four DNA mismatch repair genes: hMSH2, hMLH1, hMSH6 and hPMS2 [15]. The use of MSI-H status as a biomarker showed that the Amsterdam criteria were not specific for an inherited disorder of DNA mismatch repair deficiency [14].

Ascertainment of subjects with CRC on the basis of young age at onset led to the detection of germline mutations in DNA mismatch repair genes in subjects who did not meet the Amsterdam criteria [16]. Single cases of HNPCC may occur as the result of small family size, concealment of a positive family history, lack of disease penetrance, adoption, non-paternity or a new mutation. A strong family history of colorectal cancer therefore lacks sensitivity as well as specificity for HNPCC [17]. The Amsterdam criteria are simple and inexpensive to apply for the purposes of ascertaining high-risk families. However, it is not acceptable to equate the fulfillment of the Amsterdam criteria (or any clinical criteria) with a specific genetic disorder [18]. The unfortunate and widespread practice of defining HNPCC on the basis of meeting a limited set of clinical criteria has generated considerable confusion.

\section{Bethesda guidelines}

The demonstration of MSI-H status is highly sensitive for HNPCC but is costly and lacks specificity. This is because between 10 and $15 \%$ of sporadic colorectal cancers are MSI-H. Immunohistochemical staining for DNA mismatch repair proteins is also costly. The Bethesda guidelines were designed to limit MSI testing to cancers that would be highly likely to be HNPCC-related if found to show the MSI-H phenotype [19]. The guidelines absorbed all forms of available evidence, including histopathological criteria, in attempting to achieve the goal of a cost-effective basis for MSI testing. However the selected histological features: (1) proximally located undifferentiated CRC with a solid or cribriform architecture, and (2) signet ring cell carcinoma, were limited and based on relatively little prior validation. In a subsequent study of MSI-H CRC, there was poor inter-observer agreement on cribriforming and the feature was in fact observed more frequently in non-MSI-H CRC [20]. Furthermore, histological features in the Bethesda guidelines were redundant because they only applied to cancers presenting up to the age of 45 years when presentation up to this age was itself a recommendation for MSI testing. In a second Bethesda guideline workshop it 
was agreed that the finding of particular morphological features should serve as indication for MSI testing up to the age of 60 years (see Section 6 . below). The precise nature of the pathological features was not stated. The next section introduces the 'tumor infiltrating lymphocyte' (TIL) as the most important tissue biomarker for HNPCC.

\section{Tumor infiltrating lymphocytes}

Most investigations of the predictive value of morphological features for MSI-H status have focused on sporadic as opposed to familial CRC (HNPCC). Where the two types of MSI-H CRC have not been distinguished, sporadic MSI-H CRC will predominate. Morphological features in addition to those listed above that have been found to be discriminatory for MSI-H status include a nodular or Crohn's-like peritumoral lymphocytic reaction (mainly B lymphocytes) [21] and the presence of intra-epithelial lymphocytes (mainly cytotoxic T lymphocytes) [22]. The latter were described as tumor infiltrating lymphocytes (TILs) (Fig. 1) [22]. In a multivariate analysis based upon sporadic MSI-H CRC that included anatomical location as a variable, the most important variables were type (adenocarcinoma, mucinous carcinoma and 'medullary' or 'undifferentiated' carcinoma), proximal anatomical location, poor differentiation, and TILs [23]. TILs were scored as present or absent and were observed in only $1 \%$ of non-MSI-HCRC but in 33\% of MSI-H CRC [23]. TILs were less frequent in mucinous carcinoma (15\%), but were useful in identifying MSI-H CRC from among the non-mucinous adenocarcinomas.

Subsequent studies counted the number of TILs in either haematoxylin and eosin (H\&E) stained sections or following immunohistochemical staining for the $\mathrm{T}$ cell markers CD3 and/or CD8. In one study it was found that when the number of $\mathrm{CD} 3$ positive cells was less than 50 in an area of epithelium that included 1000 tumor and lymphocyte nuclei $(5 \%)$, the tumors were scored as TIL negative in H\&E sections [24]. TIL positive tumors were therefore defined on the basis of a cut-off score of $5 \%$ or more. The proportion of TIL positive cancers according to MSI-H status was $72 \%$ (MSI-H), 30\% (MSI-L) and 12.5\% (MSS) [24]. Based on counts of CD3 positive cells per 5 high power fields (totaling $0.94 \mathrm{~mm}^{2}$ ), a second study established a cutoff value of 8 TILs per high power field [20]. The sensitivity of $8 \mathrm{CD} 3+$ TILs per high power field for diagnosing MSI-H cancers was $75 \%$ with a specificity of $67 \%$ [20].

TILs are less easily identified in H\&E sections and somewhat lower cut-off values of 3, 0.7 and $>2$ per high power field were used in three independent studies [25-27]. In the study employing a cut-off of 0.7 TILs per high power field (which included a subset of HNPCC CRC as well as sporadic MSI-H CRC), the sensitivity and specificity of TILs for diagnosing MSI$\mathrm{H}$ status were $88 \%$ and $75 \%$ respectively [26]. In a second study that employed a population-based series of CRC with no enrichment by HNPCC, a cut-off of $>2$ TILs per high power field resulted in $90 \%$ sensitivity and $77 \%$ specificity for MSI-H [27]. The sensitivity of histological prediction of MSI-H status was increased to $100 \%$ in the last study by the addition of two further variables: (1) any amount of mucinous differentiation, and (2) absence of 'dirty necrosis' within glandular lumina. It is clear that pathological features are very sensitive for the identification of sporadic MSI-H cancers, but the somewhat lower specificity and rarity of MSI-H $\mathrm{CRC}$ in the general population means that the positive predictive value is low. Assuming that the same variables can be used to diagnose HNPCC, the positive predictive value is diminished further by the comparative rarity of HNPCC. The point has been made, however, that around $50 \%$ of $\mathrm{CRC}$ can be excluded from testing for MSI status on the basis of the rapid and inexpensive assessment of TIL counts [27]. The positive predictive value for applying the test to HNPCC could be improved by restricting the test to subjects below a certain age.

\section{Distinguishing HNPCC and sporadic MSI-H CRC}

It has often been assumed that sporadic CRC with chromosomal instability is the non-familial counterpart of CRC complicating FAP while sporadic CRC with MSI is the non-familial counterpart of CRC in HNPCC. It is likely that both assumptions are wrong or represent oversimplifications of the actual pathogenic mechanisms underlying each subtype of CRC. With respect to the familial (HNPCC) and non-familial classes of MSI-H CRC, it is a fact that the latter differ from the former in being more age-related, more common among females, having a greater predilection for the proximal colon, having a low frequency of dedifferentiation or tumor budding at the advancing tumor margin, having a lower frequency of mutation of APC, beta-catenin and 


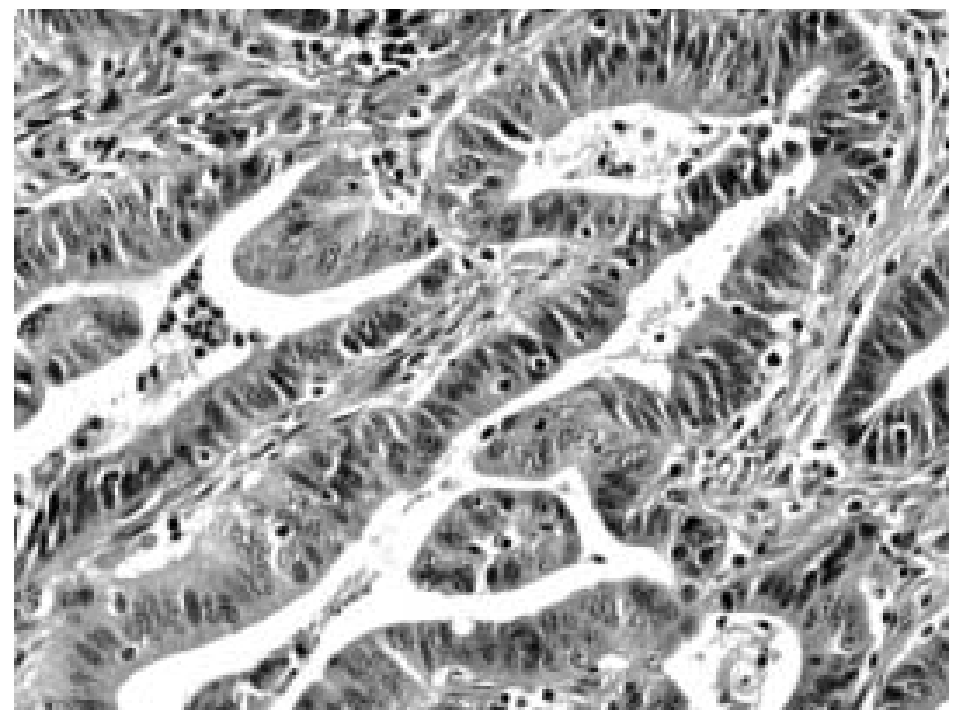

Fig. 1. Numerous tumor-infiltrating lymphocytes (TILs) in a sporadic MSI-H colorectal cancer (H\&E).

K-ras and showing a higher frequency of DNA methylation [28-30]. While there is good evidence that most HNPCC cancers develop in pre-existing adenomas [31, 32] most, if not all, sporadic MSI-H cancers develop within serrated polyps (hyperplastic-like polyps, mixed polyps and serrated adenomas) [33]. Both classes of MSI-H cancer share the feature of DNA mismatch repair deficiency. This is probably acquired at a very early evolutionary stage in HNPCC [34] while occurs at the point of transition from hyperplasia to dysplasia in the sporadic pathway [33]. Nevertheless, the shared and important step of DNA repair deficiency will result in some evolutionary convergence and therefore phenotypic overlap between the two classes of MSI-H CRC.

To what extent are the morphological features similar in HNPCC versus sporadic MSI-H CRC? It now appears that some of the generic morphological characteristics of MSI-H cancers are observed more frequently in HNPCC while others feature more strongly in sporadic MSI-H cancer. A marked mucinous component has been reported in 35\% [35], 43\% [30], 36\% [36], and $31 \%$ [25] of sporadic MSI-H cancer (Fig. 2). When a mucinous phenotype was defined on the basis of any amount of secretory mucin production, this feature was present in $67 \%$ of MSI-H cancers that were mainly sporadic [27]. On the other hand, mucinous differentiation was observed in only $19 \%$ [8] and $22 \%$ [30] of probable HNPCC cancers (not all proven by germline mutation) and in a study of 64 cancers from subjects with a proven germline mutation, mucin production was not significantly increased as compared with CRC from the general population [37]. Whilst mucinous cancers are probably slightly over-represented in HNPCC, the frequency of the finding may show inter-family differences. Mucinous carcinoma was reported in five members of a single HNPCC family [38] and was noted to be more common in families with hMSH2 as opposed to hMLH1 germline mutation, though the difference fell short of significance [37].

A CRC is mucinous merely because it recapitulates the secretory mucinous phenotype of its precursor lesion. Adenomatous polyps show loss of mucin synthesis with increasing epithelial dysplasia. Villous adenomas often show abundant mucin production and are found with increased frequency in HNPCC [31]. This would account for the slightly increased frequency of mucinous carcinoma in HNPCC. The mucinous carcinomas in HNPCC are often well differentiated and the epithelium shows a strong similarity with the epithelium of villous adenoma. The reason for the high frequency of mucin production in sporadic MSI-H CRC is also related to their histogenesis, specifically their origin within serrated polyps [33]. The latter show marked up-regulation of secretory mucin that includes both intestinal (MUC2) and gastric (MUC5AC) mucin [39]. Sporadic mucinous MSI-H CRC is often moderately to poorly differentiated, yet it may be possible to discern a serrated architectural pattern despite the loss of differentiation (Fig. 3) [30]. A particular architectural pattern found in sporadic mucinous MSI-H CRC is that of irregular chains or clusters of cells that appear to float in mucin or form lace-like or spongiform structures [20]. 


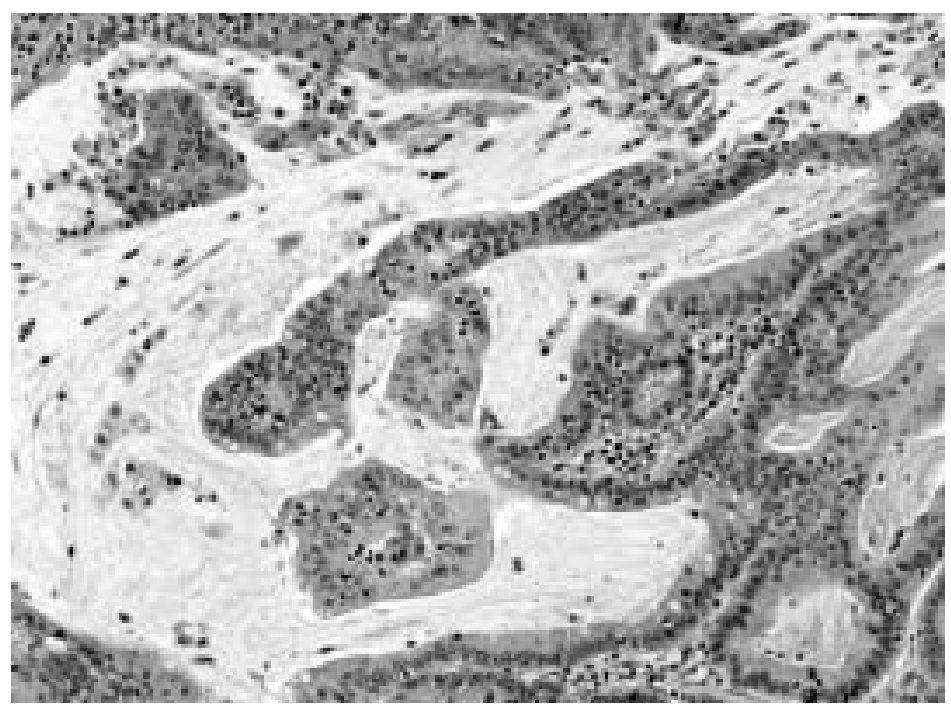

Fig. 2. Mucinous sporadic MSI-H colorectal cancer (H\&E).

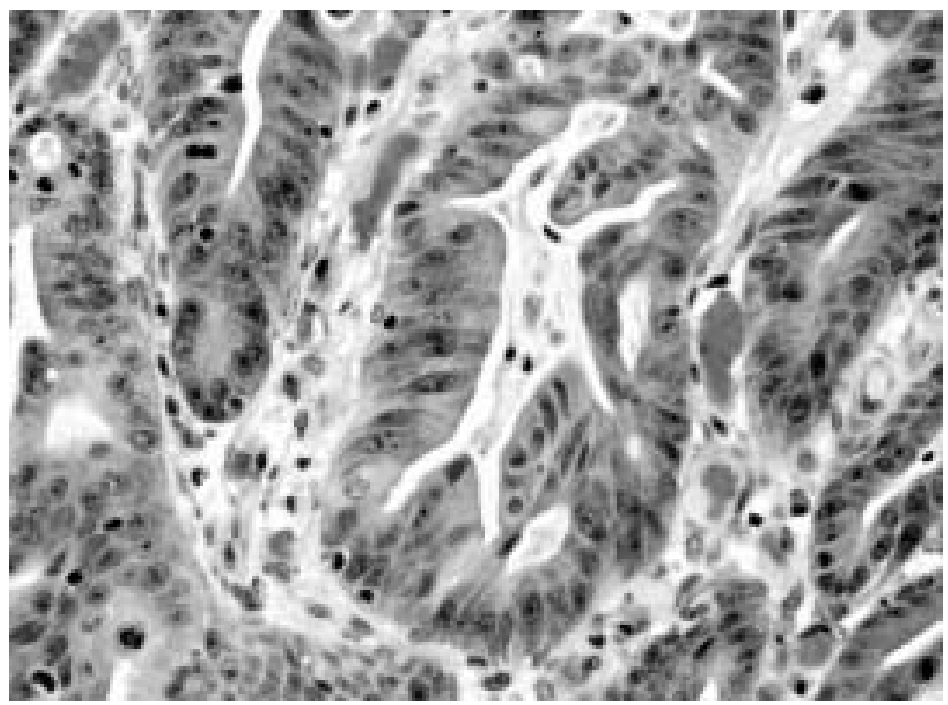

Fig. 3. Glandular serration in a sporadic MSI-H colorectal cancer (H\&E).

Sporadic MSI-H cancers are more likely to be poorly differentiated than CRC in HNPCC. There are probably two reasons for this. First is the tendency for sporadic MSI-H mucinous cancers to be poorly differentiated. Second is the greater frequency of tumor heterogeneity in sporadic MSI-H CRC [30]. If grading is based on the most poorly differentiated area, the frequent finding of poorly differentiated subclones in sporadic MSI-H $\mathrm{CRC}$ will result in many such cases being labeled as poorly differentiated. The distinctive undifferentiated or medullary pattern is over-represented in both subtypes of MSI-H CRC (Fig. 4) [9,40]. The pattern of dedifferentiation at the invasive tumor margin known as 'budding' is observed more frequently in HNPCC than sporadic MSI-H CRC [28]. The policy of labeling this alteration as poor differentiation would inflate the number of poorly differentiated CRC in HNPCC.

While CRCs in HNPCC may be less likely than sporadic MSI-H CRCs to be classified as mucinous and less likely to show poor differentiation, they are at least as likely to show increased lymphocytic infiltration. In fact, when HNPCC and sporadic MSI-H cancers were compared without using any form of detailed cell counting, the presence of TILs, peritumoral lymphocytes and 


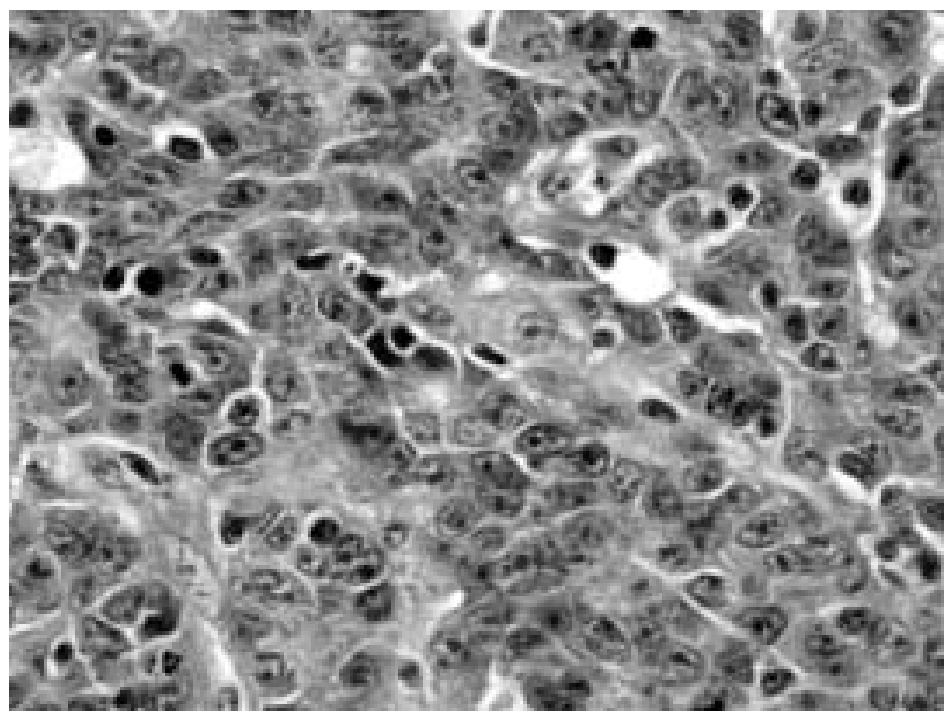

Fig. 4. Undifferentiated or medullary carcinoma from a subject with HNPCC (H\&E).

a Crohn's-like reaction was scored more frequently in HNPCC CRC, though the difference was statistically significant only for peritumoral lymphocytes [30]. This means that the detailed validation of TIL counting in sporadic MSI-H CRC will be transferable to HNPCC and will be at least as effective in identifying CRC in HNPCC as sporadic MSI-H CRC.

Whether the application of TIL counting is used to identify CRC complicating HNPCC or all CRC with MSI-H status, it is necessary to distinguish HNPCC from sporadic MSI-H CRC for the purposes of clinical management. This is generally straightforward when there is a strong family history of CRC and the other clinical manifestations of HNPCC. It is nevertheless possible for MSI-H cancers to cluster in families in which there is no germline mutation of a DNA mismatch repair gene. This could be due to familial predisposition to somatic DNA methylation and specifically to methylation of hMLH1. A clue to such a methylator background would be the finding of mutiple hyperplastic polyps, mixed polyps or serrated adenomas [41]. Additionally, there is a report of HNPCC occurring through hypermethylation of hMLH1 in the germline [42].

In the absence of family history, age at onset of CRC will be an important discriminant. A number of studies have assumed that a significant proportion of early onset MSI-H cancers are truly sporadic when there is no strong family history of CRC. There are five cogent and interrelated reasons for questioning the presumption that early onset 'sporadic' MSI-H cancers are truly sporadic (implying the lack of a germline muta- tion in a mismatch repair gene). First is the fact that the incidence of HNPCC peaks at around 45 years [6]. Second is the finding of germline mutations in DNA mismatch repair genes in subjects presenting with early onset 'sporadic' MSI-H colorectal cancer [16]. Third is the evidence that methylation and/or loss of expression of hMLH1 in sporadic MSI-H cancer is strongly age-related [43] Fourth is the fact that methylation of hMLH1 may occur selectively in HNPCC cancers in subjects who carry a germline mutation in hMLH1 [30]. Fifth is the finding of HNPCC-type molecular features such as mutation of beta-catenin amongst early onset 'sporadic' MSI-H colorectal cancers [44]. In a series of 57 sporadic MSI-H CRC, the mean age of subjects was 74.5 years and the youngest subject was aged 57 years [30]. For this reason, it is likely that most MSI-H cancers detected below the age of 60 years will be from subjects with HNPCC, regardless of the presence or absence of a family history of CRC.

\section{Practical issues}

The purpose of the Bethesda guidelines is to identify $\mathrm{CRC}$ that present in subjects with an increased probability of being affected by HNPCC for further investigation of a DNA mismatch repair defect. In the revised Bethesda guidelines, the utilization of histopathological features applies to all cancers presenting below the age of 60 years [45]. The actual features were not specified, but the data presented above indicate that a single 
A

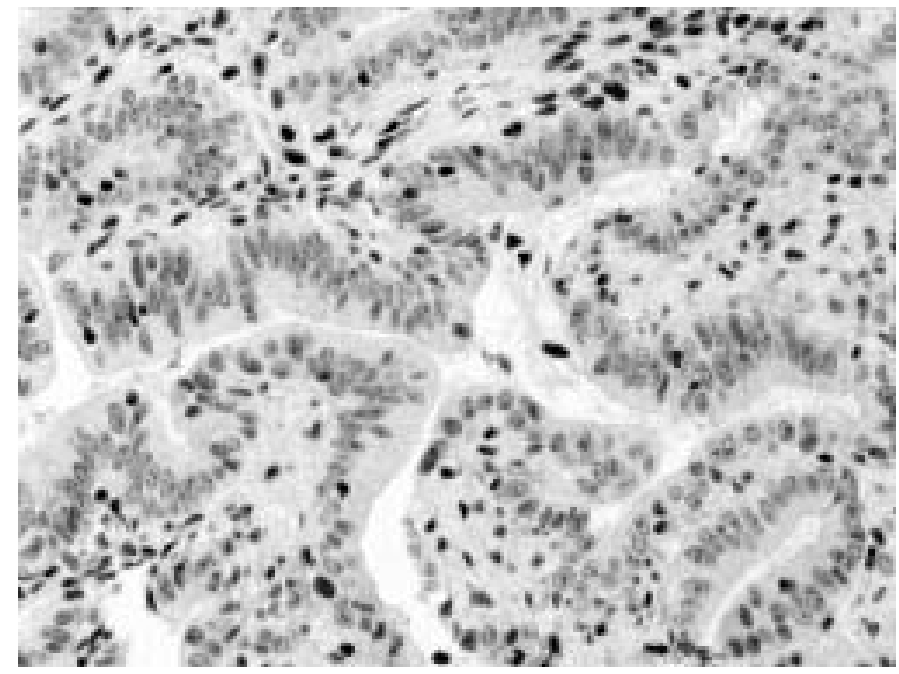

B

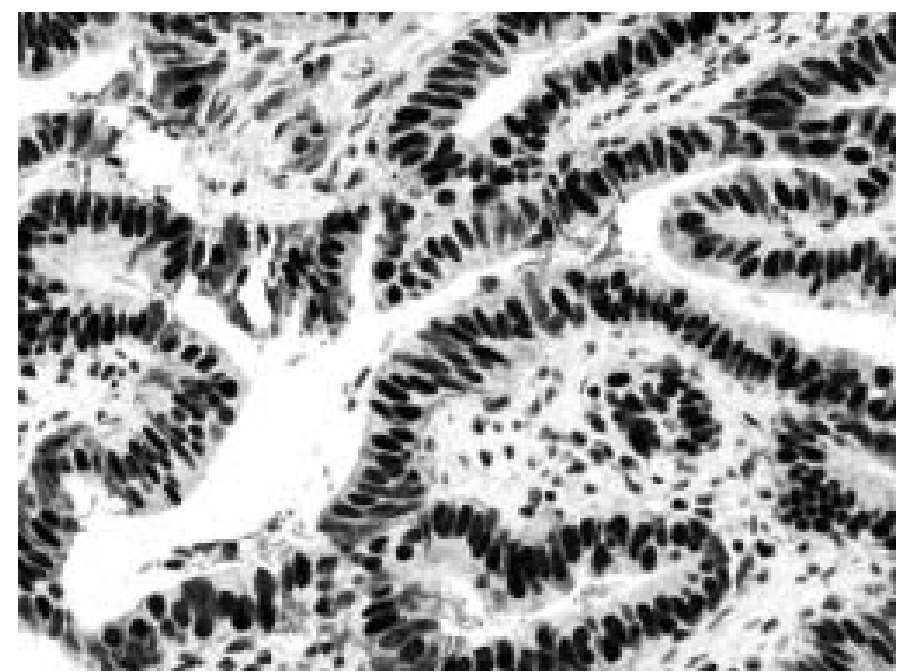

Fig. 5. Immunostaining of colorectal cancer from subject with HNPCC showing loss of expression of hMLH1 (A) and retained expression of hMSH2 (B). Tumour infiltrating lymphocytes in 5A show retained expression of hMLH1 (avidin-biotin immunoperoxidase technique).

feature may be used in the case of HNPCC cancers: tumor infiltrating lymphocytes (TILs). While medullary carcinoma is highly sensitive for HNPCC (Fig. 4), this type of cancer is associated with large numbers of TILs. To maximize sensitivity, a positive intraepithelial lymphocyte count should be 7 or more unequivocal TILs per 10 high power fields in an H\&E stained section. TILs have a slightly convoluted and dark staining nucleus with a clear halo and are easily distinguished from apoptotic epithelial cells and mitoses at high magnification (Fig. 1). TILs are normally found in colorectal epithelium and in epithelial polyps. Therefore the count should not be made in intramucosal neoplasia but only in unequivocally infiltrating carcinoma. The most effective cut-off value may vary between opera- tors who have different thresholds for recognizing an unequivocal TIL.

The majority of HNPCC CRC presents below the age of 60 years. Given the high sensitivity of TILs for HNPCC, the pathologist is now in a position to diagnose and initiate further work-up in nearly all new cases of HNPCC. The diagnosis may also be suspected on the basis of family history and young age at onset of CRC. The application of histopathological features to CRC presenting below the age of 50 years might appear redundant given the fact that age below 50 years is a criterion for further testing by itself. However, it would not be appropriate for a pathologist to ignore a positive TIL count merely because the patient was below 50 years. In fact, the combination young age at onset and 
a positive TIL count will increase the likelihood of a diagnosis of HNPCC and the combination of the two will strengthen the diagnostic safety net.

The application of immunohistochemistry may not only confirm the suspicion of DNA mismatch repair deficiency but can pinpoint the underlying mutated gene (Figs 5(A) and (b)) [46-48]. Moreover loss of expression of hMSH2 and/or hMSH6 is probably synonymous with a diagnosis of HNPCC, since most, if not all, sporadic MSI-H cancers are explained by silencing of hMLH1 [49]. Therefore the pathologist may not only suspect HNPCC, but may be able to achieve a working diagnosis of a genetic condition that will have important implications for the patient and the patient's family. According to the revised Bethesda guidelines, all CRC presenting under the age of 50 should be tested for DNA mismatch repair deficiency [45]. However, if the cancer lacks TILs and all other morphological biomarkers of DNA MSI-H status, it is questionable if MSI testing or immunohistochemistry should be undertaken.

\section{Medicolegal issues}

A pathologist is trained to provide an accurate and comprehensive diagnosis of disease on the basis of the inspection and special examination of tissues that are submitted by a clinician. Guided by the pathologist's report, the clinician will in turn be in a position to treat the patient in an effective and beneficial manner. Increasingly, the unique structure of a person's DNA has come to be viewed as a codified version of the person's integrity and autonomy. A genetic diagnosis should therefore only be reached with the consent of the individual who can then stipulate than the genetic information is confidential and limit the use to which it is put. In the case of familial cancer, it is clear that the withholding of genetic information can inflict preventable harm on multiple third parties. In the Safer case that concerned the failure to warn the members of an FAP family of their increased risk of CRC, the Appeals Court in New Jersey considered the physician to be acting as a family counselor and therefore responsible for disclosing the hereditary nature of a patient's disease to at-risk family members. The decision of the Appeals Court left open the possibility that a physician may be held liable for failing to warn a third party even in the situation in which there was insistence by the patient that the genetic information be maintained in confidence [50].
Where does this leave the pathologist encountering a TIL positive colorectal cancer in a young subject? The failure to diagnose HNPCC could in particular circumstances be the key error in the causation of preventable harm and might therefore be construed as medical negligence. One option for the pathologist would be to raise the possibility of HNPCC in the report without undertaking the step of immunohistochemistry. The clinician may then obtain the consent of the patient to undertake further investigation that would include immunohistochemistry. On the other hand, TIL counting and even the gross inspection of a colon from a subject with multiple polyps could be considered as forms of 'genetic testing'. Immunohistochemistry is a test of phenotype rather than genotype and it is not known if loss of a particular DNA mismatch repair gene is tantamount to a diagnosis of HNPCC with $100 \%$ certainty. Performing immunohistochemistry at the time of the routine diagnostic work-up is certainly more convenient for a laboratory and would probably increase the likelihood that the stated suspicion of HNPCC in a pathology report was followed up by appropriate counseling and definitive investigation of the condition. It is therefore my view that immunohistochemistry should be initiated by the pathologist but that a positive result should not be equated with a definite diagnosis of HNPCC. However, the pathologist should have discussed this policy with the clinician as the latter may prefer to obtain the consent of the patient before immunostaining is instigated.

\section{References}

[1] A.S. Warthin, Heredity with reference to carcinoma as shown by the study of the cases examined in the pathological laboratory of the University of Michigan, Arch Int Med 12 (1913), 546-555.

[2] E.D. Williams, A review of 17 cases of carcinoma of the thyroid and phaeochromocytoma, J Clin Pathol 18 (1965), 288-292.

[3] H.R. Wiedemann, Tumor and hemihypertrophy associated with Wiedemann-Beckwith's syndrome, Eur J Pediatr 141 (1983), 129-131.

[4] H.T. Lynch and A.J. Krush, Cancer family "G" revisited: 1895-1970, Cancer 27 (1971), 1505-1511.

[5] H.T. Lynch, M.D. Patrick, P.M. Lynch, J.D. William, W.A. Albano and J.F. Lynch, The cancer syndrome: a status report, Dis Colon Rectum 24 (1981), 311-322.

[6] H.T. Lynch, T.C. Smyrk and P. Watson et al., Genetics, natural history, tumor spectrum, and pathology of hereditary nonpolyposis colorectal cancer: An updated review, Gastroenterology 104 (1993), 1535-1549.

[7] H.T. Lynch, T. Smyrk and J.F. Lynch, Overview of natural history, pathology, molecular genetics and mangement of $\mathrm{HN}$ PCC (Lynch syndrome), Int J Cancer 69 (1996), 38-43. 
[8] J.R. Jass, T.C. Smyrk, S.M. Stewart, M.R. Lane, S.J. Lanspa and H.T. Lynch, Pathology of hereditary non-polyposis colorectal cancer, Anticancer Res 14 (1994), 1631-1634.

[9] J. Rüschoff, W. Dietmaier and J. Lüttges et al., Poorly differentiated colonic adenocarcinoma, medullary type: Clinical, phenotypic, and molecular characteristics, Am J Pathol 150 (1997), 1815-1825.

[10] N.M. Gibbs, Undifferentiated carcinoma of the large intestine, Histopathology 1 (1977), 77-84.

[11] G. De Petris, R. Lev, D.M. Quirk, P.R. Ferbend, J.R. Butmarc and K. Elenitoba-Johnson, Lymphoepithelioma-like carcinoma of the colon in a patient with hereditary nonpolyposis colorectal cancer, Arch Pathol Lab Med 123 (1999), 720-724.

[12] J.R. Jass, L. Constable and R. Sutherland et al., Adenocarcinoma of colon differentiating as dome epithelium of gutassociated lymphoid tissue, Histopathology 36 (2000), 116120.

[13] Y. Ionov, M.A. Peinado, S. Malkhosyan, D. Shibata and M. Perucho, Ubiquitous somatic mutations in simple repeated sequences reveal a new mechanism for colonic carcinogenesis, Nature 363 (1993), 558-561.

[14] J.R. Jass, D.S. Cottier and P. Jeevaratnam et al., Diagnostic use of microsatellite instability in hereditary non-polyposis colorectal cancer, Lancet 346 (1995), 1200-1201.

[15] H.F. Vasen, P. Watson, J.-P. Mecklin and H.T. Lynch, New clinical criteria for hereditary nonpolyposis colorectal cancer (HNPCC, Lynch syndrome) proposed by the International Collaborative Group on HNPCC, Gastroenterology 116 (1999), 1453-1456.

[16] S.M. Farrington, J. Lin-Goerke and J. Ling et al., Systematic analysis of hMSH2 and hMLH1 in young colon cancer patients and controls, Am J Hum Genet 63 (1998), 749-759.

[17] N.E. Beck, I.P.M. Tomlinson, T. Homfray, S.V. Hodgson, C.J. Harocopos and W.F. Bodmer, Genetic testing is important in families with a history suggestive of hereditary non-polyposis colorectal cancer even if the Amsterdam criteria are not fulfilled, Br J Surg 84 (1997), 233-237.

[18] M.J.W. Berends, Y. Wu and R.H. Sijmons et al., Clinical definition of hereditary non-polyposis colorectal cancer: A search for the impossible, Scand J Gastroenterol 36(234) (2001), 61-67.

[19] M.A. Rodriguez-Bigas, C.R. Boland and S.R. Hamilton et al., A National Cancer Institute Workshop on Hereditary Nonpolyposis Colorectal Cancer Syndrome: Meeting Highlights and Bethesda Guidelines, J Natl Cancer Inst 89 (1997), 17581762 .

[20] J. Alexander, T. Watanabe, W. Tsung-Teh, A. Rashid, S. Li and S.R. Hamilton, Histopathological identification of colon cancer with microsatellite instability, Am J Pathol 158 (2001), 527-535.

[21] D.M. Graham and H.D. Appelman, Crohn's-like lymphoid reaction and colorectal carcinoma: a potential histologic prognosticator, Mod Pathol 3 (1990), 332-335.

[22] M. Krishna, L.J. Burgart, A.J. French, L. Moon-Tasson, K.C. Halling and S.N. Thibodeau, Histopathologic features associated with microsatellite instability in colorectal carcinomas, Gastroenterology 110 (1996), A546.

[23] J.R. Jass, K.-A. Do and L.A. Simms et al., Morphology of sporadic colorectal cancer with DNA replication errors, Gut 42 (1998), 673-679.

[24] J.M. Michael-Robinson, A.-E. Biemer-Hüttman and D.M. Purdie et al., Tumour infiltrating lymphocytes and apoptosis are independent features in colorectal cancer stratified accord- ing to microsatellite instability status, Gut 48 (2001), 360366.

[25] R. Ward, A. Meagher and I. Tomlinson et al., Microsatellite instability and the clinicopathological features of sporadic colorectal cancer, Gut 48 (2001), 821-829.

[26] T.C. Smyrk, P. Watson, K. Kaul and H.T. Lynch, Tumorinfiltrating lymphocytes are a marker for microsatellite instability in colorectal cancer, Cancer 91 (2001), 2417-2422.

[27] J.K. Greenson, J.D. Bonner and O. Ben-Yzhak et al., Phenotype of microsatellite unstable colorectal carcinomas, Am J Surg Pathol 27 (2003), 563-570.

[28] J.R. Jass, M. Barker and L. Fraser et al., APC mutation and tumour budding in colorectal cancer, J Clin Pathol 56 (2003), 69-73.

[29] J.R. Jass, M.D. Walsh, M. Barker, L.A. Simms, J. Young and B.A. Leggett, Distinction between familial and sporadic forms of colorectal cancer showing DNA microsatellite instability, Eur J Cancer 38 (2002), 858-866.

[30] J. Young, L.A. Simms and K.G. Biden et al., Features of colorectal cancers with high-level microsatellite instability occurring in familial and sporadic settings: Parallel pathways of tumorigenesis, Am. J. Pathol. 159 (2001), 2107-2116.

[31] J.R. Jass and S.M. Stewart, Evolution of hereditary nonpolyposis colorectal cancer, Gut 33 (1992), 783-786.

[32] F.E. Rijcken, H. Hollema and J.H. Kleibeuker, Proximal adenomas in hereditary non-polyposis colorectal cancer are prone to rapid malignant transformation, Gut 50 (2002), 382-386.

[33] J.R. Jass, V.L.J. Whitehall, J. Young and B.A. Leggett, Emerging concepts in colorectal neoplasia, Gastroenterology 123 (2002), 862-876.

[34] H. Iino, L.A. Simms and J. Young et al., DNA microsatellite instability and mismatch repair protein loss in adenomas presenting in hereditary non-polyposis colorectal cancer, Gut $\mathbf{4 7}$ (2000), 37-42.

[35] H. Kim, J. Jen, B. Vogelstein and S.R. Hamilton, Clinical and pathological characteristics of sporadic colorectal carcinomas with DNA replication errors in microsatellite sequences, $A m$ J Pathol 145 (1994), 148-156.

[36] R. Gafa, I. Maestri and M. Matteuzzi et al., Sporadic colorectal adenocarcinomas with high-frequency microsatellite instability, Cancer 89 (2000), 2025-2037.

[37] M. Shashidharan, T. Smyrk and K.M. Lin et al., Histologic comparison of hereditary nonpolyposis colorectal cancer associated with MSH2 and MLH1 and colorectal cancer from the general population, Dis Colon Rectum 42 (1999), 722-726.

[38] J.R. Jass, S.M. Stewart, J. Stewart and M.R. Lane, Hereditary non-polyposis colorectal cancer: morphologies, genes and mutations, Mutat Res 290 (1994), 125-133.

[39] A.-E. Biemer-Hüttmann, M.D. Walsh and M.A. McGuckin et al., Immunohistochemical staining patterns of MUC1, MUC2, MUC4, and MUC5AC mucins in hyperplastic polyps, serrated adenomas, and traditional adenomas of the colorectum, J Histochem Cytochem 47 (1999), 1039-1047.

[40] J.R. Jass, D.S. Cottier, P. Jeevaratnam, V. Pokos and P. Browett, Pathology of hereditary non-polyposis colorectal cancer with clinical and molecular genetic correlations, in: New Strategies for Treatment of Hereditary Colorectal Cancer, Vol. (in press), S. Baba, ed., Japan: Churchill Livingstone, 1996.

[41] J.R. Jass, D.S. Cottier, V. Pokos, S. Parry and I.M. Winship, Mixed epithelial polyps in association with hereditary nonpolyposis colorectal cancer providing an alternative pathway of cancer histogenesis, Pathology 29 (1997), 28-33.

[42] I. Gazzoli, M. Loda, J. Garber, S. Syngal and R.D. Kolodner, A hereditary nonpolyposis colorectyal carcinoma case associ- 
ated with hypermethylation of the MLH1 gene in normal human tissue and loss of heterozygosity of the unmethylated allele in the resulting microsatellite instability-high tumor, Cancer Res 62 (2002), 3925-3928.

[43] S. Kakar, L.J. Burgart and S.N. Thibodeau et al., Frequency of loss of hMLH1 expression in colorectal carcinoma increases with advancing age, Cancer 2002 (2003), 1421-1427.

[44] R. Gryfe, H. Kim and E.T.K. Hsieh et al., Tumor microsatellite instability and clinical outcome in young patients with colorectal cancer, $N$ Eng J Med 342 (2000), 69-77.

[45] A. Umar, C.R. Boland, J.P. Terdiman et al., Revised Bethesda Guidelines for heriditary nonpolyposis colorectal cancer (Lynch Syndrome) and microsatellite instability, J. Natl. Cancer Inst. 96 (2004), 261-268.

[46] V.A. Marcus, L. Madlensky and R. Gryfe et al., Immunohistochemistry for hMLH1 and hMSH2: a practical test for DNA mismatch repair-deficient tumors, Am J Surg Pathol 23 (1999), 1248-1255.
[47] F. Paraf, M. Gilquin and M. Longy et al., MLH1 and $\mathrm{MSH} 2$ protein immunohistochemistry is useful for detection of hereditary non-polyposis colorectal cancer in young patients, Histopathology 39 (2001), 250-258.

[48] N.M. Lindor, L.J. Burgart and O. Leontovich et al., Immunohistochemistry versus microsatellite instability testing in phenotyping colorectal tumors, J. Clin. Oncol. 20 (2002), 1043 1048.

[49] S.N. Thibodeau, A.J. French and J.M. Cunningham et al., Microsatellite instability in colorectal cancer: Different mutator phenotypes and the principle involvement of hMLH1, Cancer Res 58 (1998), 1713-1718.

[50] H.T. Lynch, J. Paulson, M. Severin, J. Lynch and P. Lynch, Failure to diagnose hereditary nonpolyposis colorectal cancer case, Dis Colon Rectum 42 (1999), 31-35. 


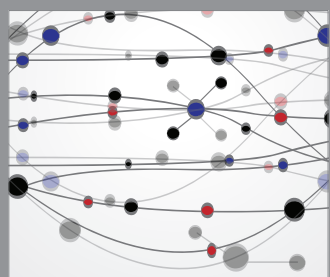

The Scientific World Journal
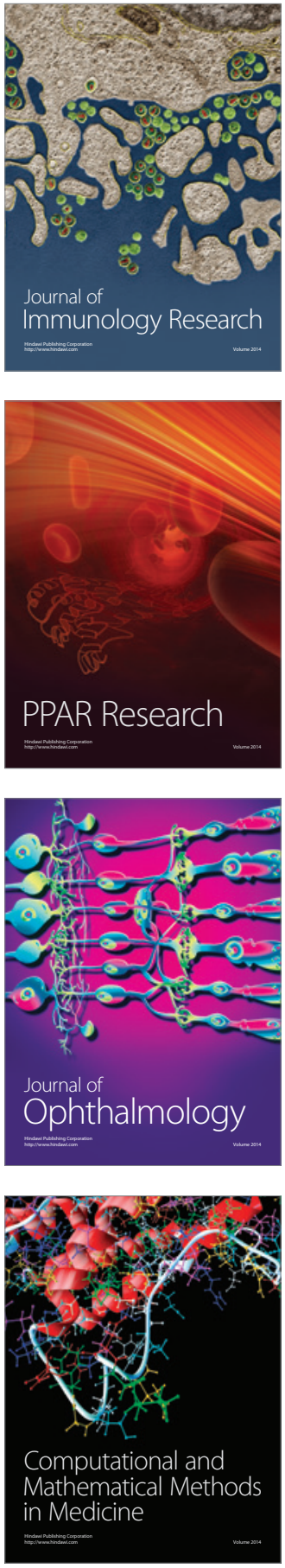

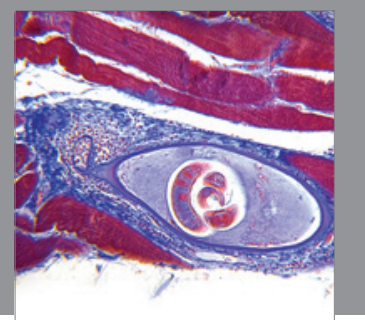

Gastroenterology

Research and Practice
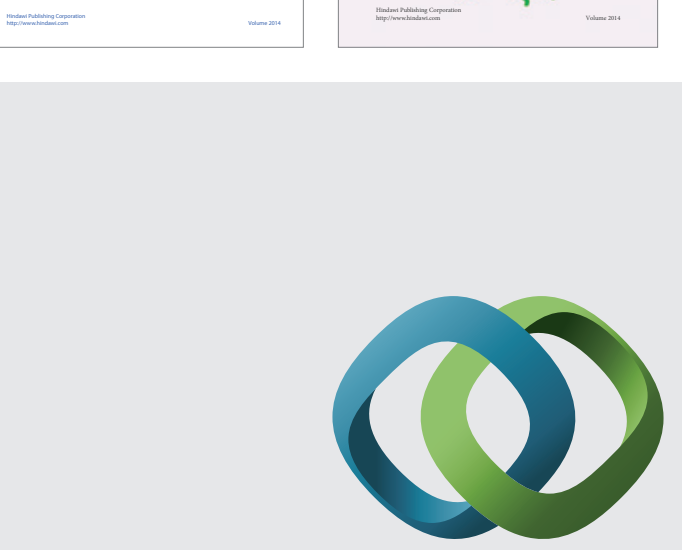

\section{Hindawi}

Submit your manuscripts at

http://www.hindawi.com
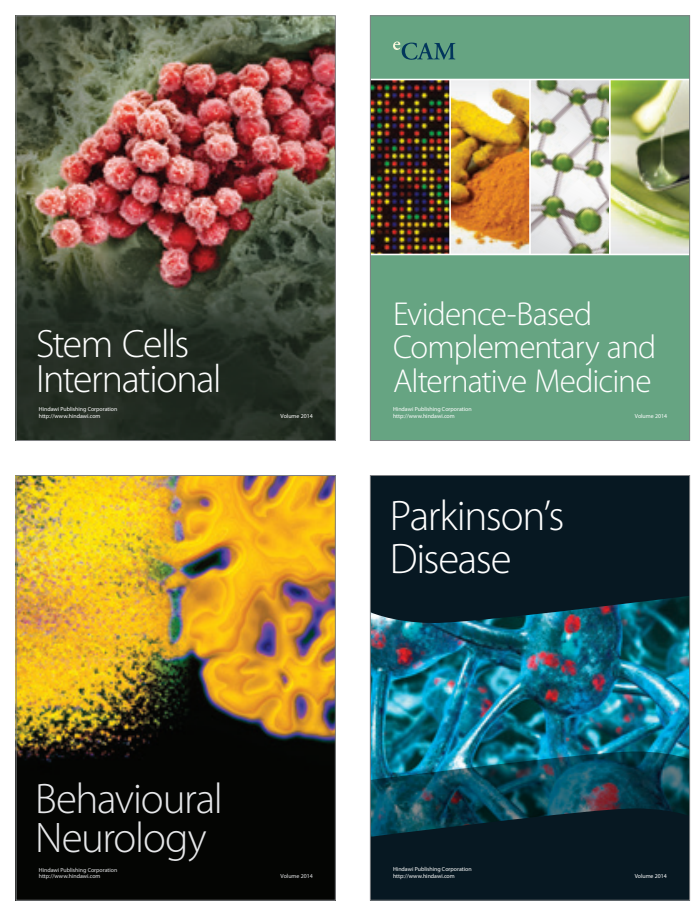

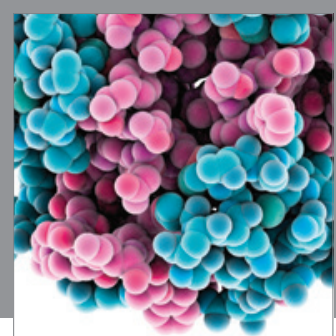

Journal of
Diabetes Research

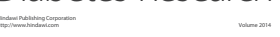

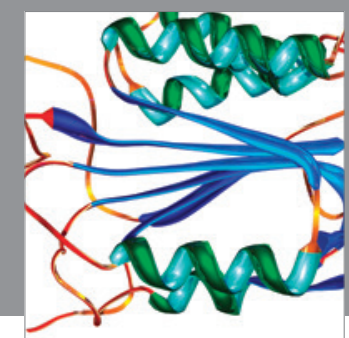

Disease Markers
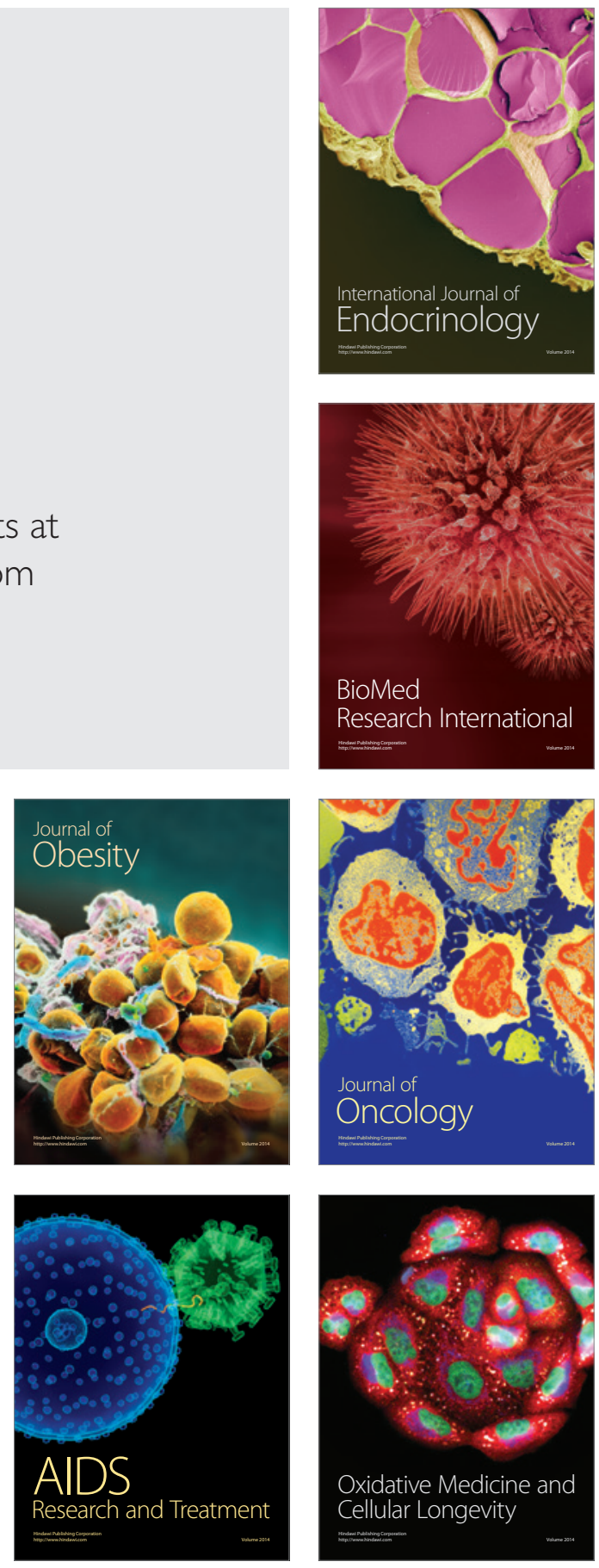